\title{
TMEM151A variants cause paroxysmal kinesigenic dyskinesia
}

\author{
Hong-Fu Li', Yu-Lan Chen', Ling Zhuang ${ }^{2,3}$, Dian-Fu Chen', Hua-Zhen Ke', Wen-Jiao Luo', Gong-Lu Liu4, \\ Sheng-Nan $\mathrm{Wu}^{5}$, Wen-Hao Zhou ${ }^{6}$, Zhi-Qi Xiong ${ }^{2,3 凶}$ and Zhi-Ying Wu (i] ${ }^{1 凶}$
}

\section{Dear Editor,}

Paroxysmal kinesigenic dyskinesia (PKD) (MIM $128200)$ is an autosomal dominant movement disorder characterized by involuntary movements which are usually triggered by sudden movements. We previously identified PRRT2 as the first causative gene of $\mathrm{PKD}^{1}$, which was widely verified in different populations ${ }^{2-4}$. It is estimated that PRRT2 variants account for $77 \%-93 \%$ familial PKD and $21 \%-45 \%$ isolated $P_{K D}^{5}$. A significant percentage of PRRT2-negative PKD patients indicate that other genes are implicated in PKD. Here, we performed whole-exome sequencing (WES) in 5 PKD pedigrees and 31 isolated PKD patients without PRRT2 variants. Moreover, 1000 unrelated healthy individuals of matched ethnicity were included as control subjects. This study was approved by the local Ethics Committee. All participants or their guardians provided written informed consents.

To systematically identify the causative genes for these PRRT2-negative PKD patients, we first analyzed three PKD families with multiple patients and unaffected individuals. Based on the autosomal dominant inheritance pattern in these families, we prioritized the heterozygous nonsynonymous variants in coding regions and splicing sites, with a minor allele frequency (MAF) $<0.01 \%$ in the genome Aggregation Database (gnomAD), and absent in the 1000 Genomes Project (1000G), Exome Sequencing

\footnotetext{
Correspondence: Zhi-Qi Xiong (xiongzhiqi@ion.ac.cn) or Zhi-

Ying Wu (zhiyingwu@zju.edu.cn)

${ }^{1}$ Department of Neurology and Research Center of Neurology in Second

Affiliated Hospital, Zhejiang University School of Medicine, and Key Laboratory of Medical Neurobiology of Zhejiang Province, Hangzhou, Zhejiang, China

${ }^{2}$ Institute of Neuroscience and State Key Laboratory of Neuroscience, CAS Center for Excellence in Brain Science and Intelligence Technology, Chinese Academy of Sciences, Shanghai, China

Full list of author information is available at the end of the article

These authors contributed equally: Hong-Fu Li, Yu-Lan Chen, Ling Zhuang
}

Project v.6500 (ESP6500), Exome Aggregation Consortium (ExAC). Besides, variants should be shared by all affected individuals but not by the unaffected parent of the proband in each family. After filtering, we found 11 candidate genes in Family 1, 29 in Family 2, and 52 in Family 3 (Supplementary Table S1). The comparison of these candidate genes revealed that TMEM151A (NM_153266) was the only gene implicated in these families. Three TMEM151A variants including c.1275dupG (p. P426Afs*19), c.375 C > A (p.C125X), and c.758 T > C (p. L253P) were confirmed by Sanger sequencing and cosegregation analysis (Fig. 1a). We did not find any TMEM151A variant in the other two PKD families. The detailed WES data are shown in Supplementary Table S2. We then screened TMEM151A variants in the WES data of 31 isolated PKD patients. We found four truncated variants (c.7 G > T [p.E3X], c.623_624insA [p. L210Afs*136], c.739 G > T [p.E247X], and c.897_912del [p. L300Pfs"118]), three missense variants $(\mathrm{c} .140 \mathrm{~T}>\mathrm{C}$ [p. L47P], c.863 T > C [p.F288S], c.889 T > A [p.S297T]), and a non-frameshift deletion (c.142_153del [p.48_51delLTLL]) in 8 index patients (Fig. 1b and Supplementary Table S3).

To evaluate the frequency of identified TMEM151A variants in the general population, we performed Sanger sequencing in 1000 control individuals. Totally, we found four missense variants and three synonymous variants, which were predicted to be benign by bioinformatic software (Supplementary Fig. S1 and Table S4). None of the TMEM151A variants identified in PKD patients was found in controls. We then screened TMEM151A rare variants $(\mathrm{MAF}<1 \%)$ in gnomAD and found 115 damaging missense variants, 6 truncated variants, and 1 in-frame deletion in 792 individuals. There was a significant enrichment of potentially pathogenic TMEM151A variants in PKD patients, compared with the gnomAD database $(11 / 36$ vs $792 / \sim 76,000, P=8.05 \times 10^{-14}$, Supplementary Table S5). In 


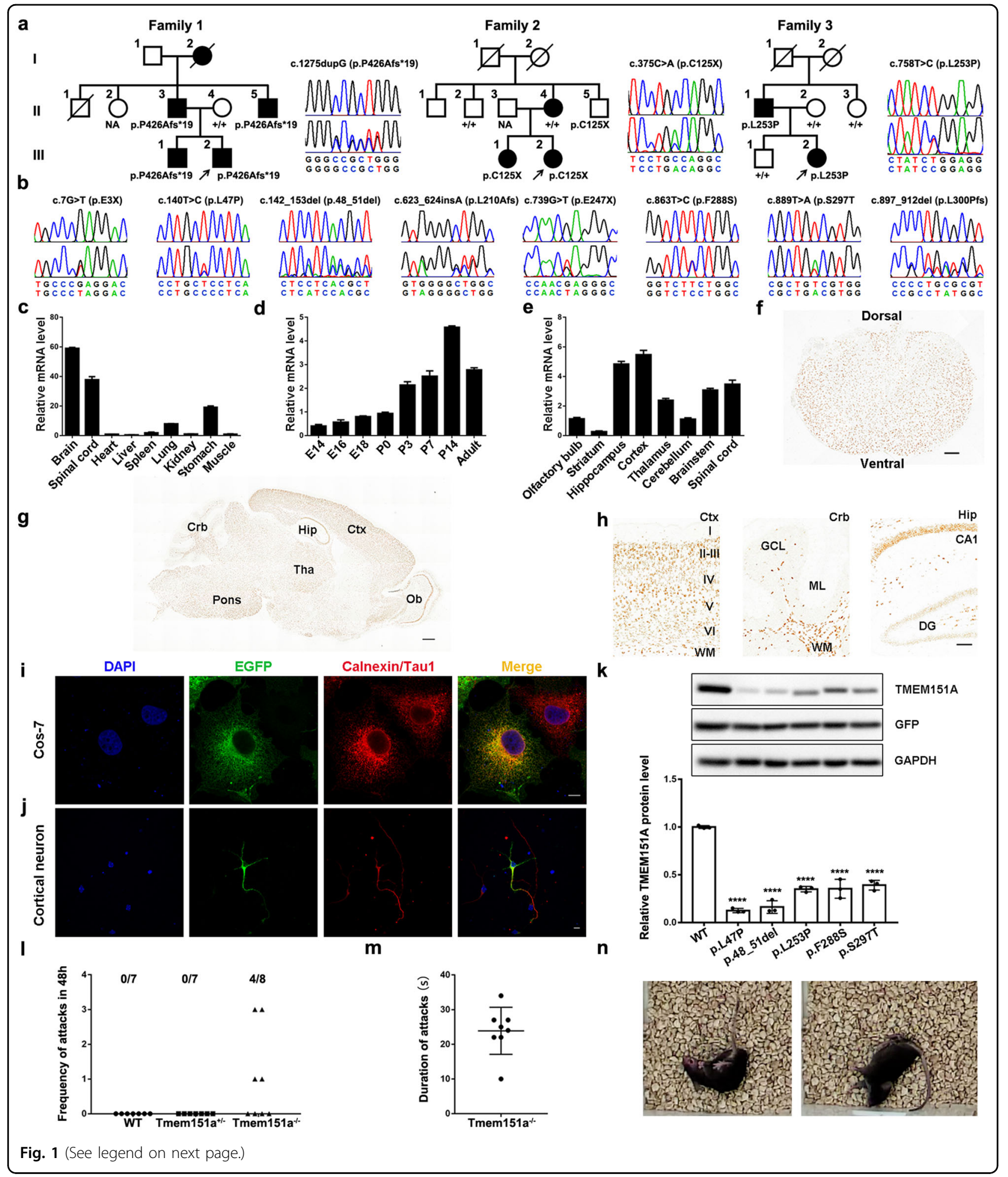

total, we identified 11 TMEM151A variants in 3 PKD pedigrees and 8 isolated patients, whose detailed clinical features are summarized in Supplementary Tables S6 and S7.
TMEM151A is a poorly characterized gene whose function is largely unknown. It is highly conserved among species (Supplementary Fig. S2). To explore its expression pattern, we measured the level of mice Tmem151a mRNA 
(see figure on previous page)

Fig. 1 TMEM151A variants cause paroxysmal kinesigenic dyskinesia. a The pedigree structure and segregation analyses of Families 1-3 and sequencing chromatograms of the identified TMEM151A variants. The upper chromatogram represents the normal sequence, and the lower represents the variant. Square: male; circle: female; arrow: index patient; filled symbol: affected; open symbol: unaffected; +: wild-type allele. b Sequencing chromatograms of TMEM151A variants identified in eight isolated PKD patients. c Relative expression level of Tmem151a mRNA in various organs of 2-month-old mice. The expression level in the heart was used as a calibration (means $\pm \mathrm{SEM}, n=3$ ). $\mathbf{d}$ Relative expression level of Tmem151a mRNA in the developing mouse brain. The expression level in PO was used as a calibration (means \pm SEM, $n=3$ ). e Relative expression level of Tmem151a mRNA in different regions of the central nervous system. The expression level in the olfactory bulb was used as a calibration (means \pm SEM, $n=3$ ). $\mathbf{f}$ In situ hybridization for Tmem151a in the P14 mouse spinal cord. Scale bar, $200 \mu \mathrm{m}$. $\mathbf{g}$, $\mathbf{h}$ In situ hybridization for Tmem $151 a$ in the P14 mouse brain. Ctx cortex, Hip hippocampus, Ob olfactory bulb, Crb cerebellum, Tha thalamus, DG dentate gyrus, ML molecular layer, GCL granule cell layer, WM white matter. Roman numerals (I-VI) indicate layers of the cerebral cortex. Scale bar, $500 \mu \mathrm{m}$ in $\mathbf{g}, 100 \mu \mathrm{m}$ in $\mathbf{h}$. i, j Fluorescence images of COS-7 cells (i) and primary cortical neurons (j) transfected with EGFP-tagged Tmem151a and immunostained with antibodies against GFP (green) and either the ER marker calnexin (red) or anti-Tau1 antibodies (axon marker, red). DAPI (blue) was used for nuclear staining. Scale bar, $10 \mu \mathrm{m}$. $\mathbf{k}$ Western blots of protein obtained from HEK293T cells transfected with WT and mutant pIRES2-Flag-TMEM151A plasmids. The anti-Flag antibody was used to detect the TMEM151A protein. The GFP was used to measure the transfection efficiency and served as an internal control. Bar graph shows protein expression level. Data are presented as means $\pm S D, n=3$, ****P $<0.0001$. I The number of dyskinesia attacks in WT, heterozygous

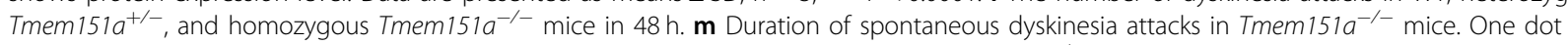
represents one observed attack. $\mathbf{n}$ Representative images of dyskinesia attacks in a Tmem151 ${ }^{-1-}$ mouse.

by real-time PCR. We found Tmem151a was highly expressed in the central nervous system (CNS), including the brain and spinal cord, followed by the stomach (Fig. 1c). Tmem151a was relatively low during the embryonic period, markedly increased during postnatal stages, peaked at postnatal day 14 (P14), and remarkably declined in adulthood (Fig. 1d). Real-time PCR and in situ hybridization analyses in P14 mice revealed that Tmem151a was ubiquitously expressed in the CNS, with a high level in the cerebral cortex, hippocampus, spinal cord, brainstem, and thalamus (Fig. 1e-g). On a more granular level, Tmem151a was enriched in cortical layers of the cerebral cortex and CA1 of the hippocampus (Fig. 1h). In the cerebellum, Tmem151a was mainly expressed in white matter, not in granule cells or Purkinje cell layers (Fig. 1h).

To examine the subcellular localization of Tmem151a protein, we generated EGFP-tagged wild-type (WT) Tmem151a plasmids and transfected them into COS-7 cells and cortical neurons. We found Tmem151a colocalized with endoplasmic reticulum (ER) marker Calnexin in COS-7 cells (Fig. 1i) and distributed in both axons and dendrites in primary cortical neurons (Fig. 1j). To elucidate the pathogenicity of TMEM151A non-truncated variants (p.L47P, p.48_51delLTLL, p.L253P, p.F288S, and p.S297T), we investigated the alternation of subcellular localization and protein expression of mutant TMEM151A. Cos-7 cells were transfected with WT and mutant EGFP-TMEM151A plasmids. Immunostaining revealed that mutant TMEM151A protein still retained on ER (data not shown). We then quantitatively measured the protein expression level of these TMEM151A variants by transfecting WT and mutant pIRES2-FlagTMEM151A plasmids into HEK 293T cells. Western blot showed a significantly decreased protein expression of mutant TMEM151A compared to WT TMEM151A
(Fig. 1k), suggesting a potential loss of function mechanism for these mutant TMEM151A.

We further generated Tmem151a knockout mice by CRISPR/Cas9-mediated genome editing according to our

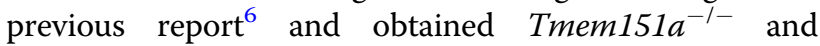
Tmem $151 a^{+l-}$ mice by breeding. Spontaneous dyskinesia was observed in both founder mice and F1 Tmem151a ${ }^{-1-}$ mice. Within $48 \mathrm{~h}$, eight times of dyskinesia attacks were observed in four out of eight 1-month-old Tmem151a ${ }^{-1-}$ mice (Fig. 11). No dyskinesia attacks were observed in Tmem $151 a^{+/-}$mice and WT mice. The duration of episodes ranged from 10 to $37 \mathrm{~s}$ (Fig. $1 \mathrm{~m}$ ). After dyskinesia attacks (Fig. 1n and Supplementary Movie S1), the mice recovered to normal locomotion.

PKD is a hereditary disorder with autosomal dominant inheritance. However, a significant proportion of PKD patients seem to be sporadic ${ }^{7}$. Incomplete penetrance, de novo mutagenesis or autosomal recessive inheritance may account for this phenomenon. In this study, we found TMEM151A variants in three autosomal dominant families and eight isolated patients. Variants c.140 T > C, c.739 G > $\mathrm{T}$, and c.623_624insA identified in three isolated patients were derived from one of their parents, who reported no obvious kinesigenic attacks. DNA samples were not available in the parents of the remaining five patients. We conjecture TMEM151A variants may have decreased penetrance, which has been observed in PRRT2 and genes responsible for dystonia ${ }^{8,9}$. Besides, TMEM151A variants could also be de novo in isolated patients.

The potential mechanisms underlying PKD are not entirely clear. The identification of $P R R T 2$ as the first causative gene of PKD has improved our understanding of the pathogenesis of the disease. Recent studies indicate that PRRT2 acts on the presynaptic terminal and plays an important role in regulating synaptic transmission and neuronal excitability ${ }^{10,11}$. TMEM151A is predicted to be a 
transmembrane protein. We found TMEM151A was localized at ER in COS-7 cells. It is known that ER is the main intracellular $\mathrm{Ca}^{2+}$ store and plays a crucial role in intracellular $\mathrm{Ca}^{2+}$ mobilization and dynamics ${ }^{12}$. Considering the paroxysmal feature of PKD, we surmise TMEM151A may be an ER-associated $\mathrm{Ca}^{2+}$ channel. Alternatively, TMEM151A may interact with $\mathrm{Ca}^{2+}$ sensors and endow the SNARE complex, like the PRRT2 protein $^{13}$. Given that patients with TMEM151A variants also obtain significant remission after carbamazepine treatment, it is possible that TMEM151A is an ion channel protein. Whether TMEM151A acts like PRRT2 in modulating $\mathrm{Na}^{+}$ channel is unclear ${ }^{14}$, which should be elucidated in the future. Loss of function might be the potential mechanism of mutant TMEM151A causing PKD. The decreased protein expression of non-truncated variants was in consistent with the mechanisms of haploinsufficiency. How amino acid residue changes affect TMEM151A protein functions requires further investigation.

In summary, we identified TMEM151A variants in both familial and isolated PKD patients, indicating that TMEM151A variants cause PKD. Although the function of TMEM151A remains elusive, we believe our findings will deepen the understanding of the mechanisms of PKD.

\section{Acknowledgements}

This work was supported by grants from the National Natural Science Foundation of China (81330025 to Z.-Y.W., 81500973 to H.-F.L.), Chinese Academy of Science (XDB02020007, QYZDJ-SSW-SMC010 to Z.-Q.X.), and the research foundation for the distinguished scholar of Zhejiang University (188020-193810101/089 to Z.-Y.W.). We thank XiangYin Biotechnology Co. Ltd and Genergy Biotechnology Inc. for assistance in WES analysis.

\section{Author details}

'Department of Neurology and Research Center of Neurology in Second Affiliated Hospital, Zhejiang University School of Medicine, and Key Laboratory of Medical Neurobiology of Zhejiang Province, Hangzhou, Zhejiang, China. ${ }^{2}$ Institute of Neuroscience and State Key Laboratory of Neuroscience, CAS Center for Excellence in Brain Science and Intelligence Technology, Chinese Academy of Sciences, Shanghai, China. ${ }^{3}$ University of Chinese Academy of Sciences, Beijing, China. ${ }^{4}$ Department of Neurology, Huashan Hospital, Shanghai Medical College, Fudan University, Shanghai, China. ${ }^{5}$ Laboratory for Molecular Diagnostics, Shanghai Children's Hospital, Shanghai Jiao Tong University, Shanghai, China. ${ }^{6}$ Department of Neonatology, Children's Hospital, Fudan University, Shanghai, China

\section{Author contributions}

Z.-Y.W. conceived and supervised the project. Z.-Q.X. supervised the project. H.F.L. and Y.-L.C. collected clinical data, performed sequencing and experiments, and wrote the manuscript. L.Z. performed experiments and wrote the manuscript. D.-F.C. and H.-Z.K. performed sequencing. W.-J.L., G.-L.L., S.-N.W., and W.-H.Z. collected clinical data.

\section{Data availability}

The original data that support the findings are available from the corresponding author (Zhi-Ying $\mathrm{Wu}$ ) on reasonable request.

\section{Conflict of interest}

The authors declare no competing interests.

\section{Publisher's note}

Springer Nature remains neutral with regard to jurisdictional claims in published maps and institutional affiliations.

Supplementary information The online version contains supplementary material available at https://doi.org/10.1038/s41421-021-00322-w.

Received: 10 March 2021 Accepted: 4 August 2021

Published online: 13 September 2021

\section{References}

1. Chen, W. J. et al. Exome sequencing identifies truncating mutations in PRRT2 that cause paroxysmal kinesigenic dyskinesia. Nat. Genet. 43, 1252-1255 (2011).

2. Wang, J. L. et al. Identification of PRRT2 as the causative gene of paroxysmal kinesigenic dyskinesias. Brain 134, 3493-3501 (2011).

3. Lee, $\mathrm{H}$. Y. et al. Mutations in the gene PRRT2 cause paroxysmal kinesigenic dyskinesia with infantile convulsions. Cell Rep, 1, 2-12 (2012).

4. Meneret, A. et al. PRRT2 mutations: a major cause of paroxysmal kinesigenic dyskinesia in the European population. Neurology 79, 170-174 (2012).

5. De Gusmao, C. M. \& Silveira-Moriyama, L. Paroxysmal movement disorderspractical update on diagnosis and management. Expert Rev. Neurother. 19, 807-822 (2019)

6. Zuo, E. et al. One-step generation of complete gene knockout mice and monkeys by CRISPR/Cas9-mediated gene editing with multiple sgRNAs. Cell Res. 27, 933-945 (2017).

7. Huang, X. J. et al. The Phenotypic and genetic spectrum of paroxysmal kinesigenic dyskinesia in China. Mov. Disord. 35, 1428-1437 (2020).

8. van Vliet, R. et al. PRRT2 phenotypes and penetrance of paroxysmal kinesigenic dyskinesia and infantile convulsions. Neurology 79, 777-784 (2012).

9. Lohmann, K. \& Klein, C. Genetics of dystonia: what's known? What's new? What's next? Mov. Disord. 28, 899-905 (2013).

10. Tan, G. H. et al. PRRT2 deficiency induces paroxysmal kinesigenic dyskinesia by regulating synaptic transmission in cerebellum. Cell Res. 28, 90-110 (2018).

11. Coleman, J. et al. PRRT2 regulates synaptic fusion by directly modulating SNARE complex assembly. Cell Rep. 22, 820-831 (2018).

12. Berridge, M. J., Bootman, M. D. \& Roderick, H. L. Calcium signalling: dynamics, homeostasis and remodelling. Nat. Rev. Mol. Cell Biol. 4, 517-529 (2003).

13. Valente, P. et al. PRRT2 is a key component of the $\mathrm{Ca}^{2+}$-dependent neurotransmitter release machinery. Cell Rep. 15, 117-131 (2016).

14. Fruscione, F. et al. PRRT2 controls neuronal excitability by negatively modulating $\mathrm{Na}^{+}$channel 1.2/1.6 activity. Brain 141, 1000-1016 (2018). 